\title{
Laboratory Investigations on Field Gas Huff-n-Puff for Improving Oil Recovery in Eagle Ford Shale -Effect of Operating Conditions
}

Chao-yu Sie, Quoc P. Nguyen*

Hildebrand Department of Petroleum and Geosystems Engineering, The University of Texas at Austin, CPE 5.104A, 200 E. Dean Keeton Stop C0300, Austin, TX 78712-1585.

*Corresponding Author

Email: quoc_p_nguyen@mail.utexas.edu

\section{Supporting Materials}

The parameters of the Peng-Robinson equation of state (EoS) are shown in Table S1 and Table S2. These parameters were determined in a comprehensive PVT study carried out and provided to us the operator. It was noted that the constant mass expansion and swelling tests conducted with the same injection gas and crude oil were used to obtain the EoS parameters in Table S1. The vapor pressure curves of the 4 pseudo components used in the model are shown in Figure S1.

Table S1. Parameters of the PR-EOS Model

\begin{tabular}{ccccccc}
\hline Comp. & $\begin{array}{c}\text { Molar } \\
\text { concentration } \\
(\mathrm{mol} \%)\end{array}$ & $\begin{array}{c}\text { Molecular } \\
\text { Weight } \\
(\mathrm{lb} / \mathrm{lb}-\mathrm{mol})\end{array}$ & $\mathrm{Tc}\left({ }^{\circ} \mathrm{F}\right)$ & $\mathrm{Pc}(\mathrm{psia})$ & $\begin{array}{c}\text { Omega } \\
(-)\end{array}$ & $\begin{array}{c}\text { Volume } \\
\text { shift } \\
\left(\mathrm{ft}^{3} / \mathrm{lbmol}\right)\end{array}$ \\
\hline $\mathrm{CH}_{4}$ & 41.967 & 16.040 & -116.6 & 667.176 & 0.00800 & -0.0833 \\
$\mathrm{C}_{2} \mathrm{H}_{6}$ & 11.855 & 30.070 & 90.05 & 708.367 & 0.09800 & -0.0927 \\
$\mathrm{C}_{3} \mathrm{H}_{8}$ & 7.661 & 44.100 & 205.97 & 615.832 & 0.15200 & -0.1017 \\
$\mathrm{C}_{4} \mathrm{H}_{10}$ & 4.278 & 58.120 & 305.69 & 551.145 & 0.19300 & -0.1017 \\
$\mathrm{C}_{5} \mathrm{H}_{12}$ & 3.040 & 72.150 & 385.61 & 489.359 & 0.25100 & -0.0820 \\
$\mathrm{C}_{6} \mathrm{H}_{14}$ & 3.909 & 86.180 & 453.65 & 430.619 & 0.29600 & 0.0223 \\
$\mathrm{PCl}$ & 11.392 & 150.195 & 725.90 & 354.584 & 0.16172 & -0.0393 \\
\hline
\end{tabular}




\begin{tabular}{crrrrrr}
\hline PC2 & 7.898 & 230.769 & 935.14 & 274.380 & 0.26048 & -0.0934 \\
PC3 & 5.250 & 354.840 & 1183.5 & 213.266 & 0.39627 & -0.1592 \\
PC4 & 2.750 & 682.480 & 1677.7 & 155.711 & 0.64461 & -0.2985 \\
\hline
\end{tabular}

Table S2. Binary Interaction Parameters for the PR-EOS Model

\begin{tabular}{cccccc}
\hline & $\mathrm{CH}_{4}$ & $\mathrm{C}_{2} \mathrm{H}_{6}$ & $\mathrm{C}_{3} \mathrm{H}_{8}$ & $\mathrm{C}_{4} \mathrm{H}_{10}$ & $\mathrm{C}_{5} \mathrm{H}_{12}$ \\
\hline $\mathrm{CH}_{4}$ & & & & \\
$\mathrm{C}_{2} \mathrm{H}_{6}$ & 0.04200 & & & & \\
$\mathrm{C}_{3} \mathrm{H}_{8}$ & 0.04200 & 0.04000 & & & \\
$\mathrm{C}_{4} \mathrm{H}_{10}$ & 0.04200 & 0.04000 & 0.03000 & & \\
$\mathrm{C}_{5} \mathrm{H}_{12}$ & 0.04200 & 0.04000 & 0.03000 & 0.01171 & \\
$\mathrm{C}_{6} \mathrm{H}_{14}$ & 0.04200 & 0.04000 & 0.03000 & 0.01568 & 0.00585 \\
$\mathrm{PC}_{1}$ & 0.05270 & 0.04171 & 0.03335 & 0.04522 & 0.03166 \\
$\mathrm{PC}_{2}$ & 0.05810 & 0.04237 & 0.03778 & 0.05936 & 0.05212 \\
$\mathrm{PC} 3$ & 0.06620 & 0.04336 & 0.04803 & 0.07021 & 0.07151 \\
$\mathrm{PC} 4$ & 0.08690 & 0.04589 & 0.07057 & 0.08081 & 0.09467 \\
\hline
\end{tabular}

* All the other values which are not presented in this able are zero.

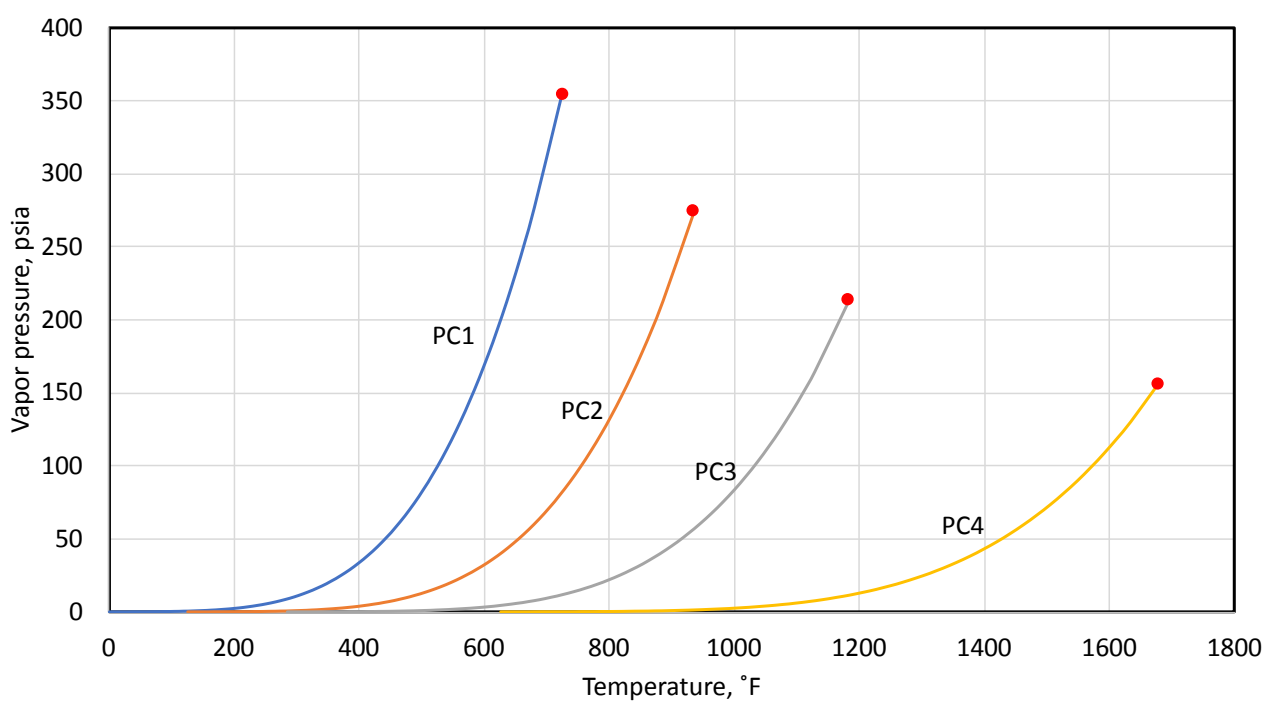

Figure S1. Vapor pressure curves of the four pseudo components. 